\title{
4
}

\section{A Roadmap for Requirements Elicitation of Knowledge Management Systems: A Delphi Study}

\author{
Sanath Sukumaran ${ }^{1}$, Hemalatha Ramalingam ${ }^{1}$, \\ Lorna Uden ${ }^{2}$, Akmal Rahim ${ }^{1}$ and Kanchana Chandran ${ }^{3}$ \\ ${ }^{1}$ Taylor's University \\ ${ }^{2}$ Staffordshire University \\ ${ }^{3}$ Freelance \\ ${ }^{3}$ Malaysia \\ ${ }^{2} U K$
}

\section{Introduction}

The discipline of Knowledge Management (KM) has been around for quite some time. However, it has yet to find a successful formula of assimilation to benefit organizations today. Organisations are not able to leverage on its past successes, experiences and lessons learnt without an effective mode to capture tacit requirements. Knowledge Management bridges this gap.

An organization's continued maturity is assessed not only for its financial strength but from its ability to leverage and build upon mainly its tacit and at times explicit nature of its intellectual capital whilst enabling co-creation of values (Lee \& Choi, 2003). To manage both tacit and explicit knowledge in an organisation, it is imperative that a Knowledge Management System (KMS) be deployed.

\section{Problem statement}

KMS requires a synergetic effort from an array of disciplines and perspectives armed with the correct technological tools to be the driving force towards a knowledge-sharing environment. Any KMS ought to look into the needs and demands of its people including cultural and organizational norms should it live up to its expectations and survive the test of time.

Software engineering as a body of knowledge does not encapsulate people, cultural and organizational facets in a holistic sense within its software development processes. Herein lies the gap.

\section{Research question}

To explore how knowledge can be elicited to suit the needs of its stakeholder taking into account cultural and organizational norms. 


\section{Research objectives}

- To review weaknesses of present KMS in achieving its intended goal.

- To ascertain how KMS differ from conventional systems to warrant a different approach.

- To propose a roadmap for requirements elicitation for Knowledge Management Systems using Activity Theory

\section{Literature review}

\subsection{Overview}

KM emerged as a scientific discipline in the early 1990s. It was initially supported solely by practitioners, when Scandia hired Leif Edvinsson of Sweden as the world's first Chief Knowledge Officer (CKO). Hubert Saint-Onge started investigating various sides of KM long before that.

The idea of a KMS is to enable employees to have ready access to the organization's documented base of facts, sources of information, and solutions. For example, an engineer could know the metallurgical composition of an alloy that reduces sound in gear systems. Sharing this information organization wide can lead to more effective engine design and it could also lead to ideas for new or improved equipment. (Langton \& Robbins, 2006).

\subsection{Knowledge management systems}

It has been suggested that the term "KMS project" should never be used as if it pertained to the same category as an IT or IM project (Terra \& Gordon, 2002). KMS projects should take a holistic or organic view of the enterprise and should encompass different initiatives in many areas: certainly in IM, but also in HR, organizational design, internal communications and so forth. KM is more closely associated with the "act of managing" than IM.

In this sense, KM is never-ending. It is defined by the identification of people's expertise and the interplay of people with people (tacit knowledge-sharing) and people with information systems (two-way road of knowledge capture, reuse and recreation). Given that they are highly dependent on people's previous knowledge, motivation and willingness to create, act, share and or codify their own individual knowledge, KM processes are far more complex than IM projects. However, KMS is increasingly dependent on the support of a solid IT infrastructure.

\subsection{Activity theory}

Initial large-scale software development efforts were chaotic, often resulting in an explosion of costs and development times which were much larger than originally predicted. The outcome was typically quite different from the original objective of the activity. Many times the object was simply not produced and the effort was abandoned after the investment of considerable resources, (Barthelmess \& Anderson, n.d).

As a result, practitioners turned their attention to the development process itself. A new term, software engineering, was coined to represent this endeavour. Software engineering strives to further define techniques, processes, methodologies, and languages to ease the development of large software systems, (Barthelmess \& Anderson, n.d).

Software engineering approaches the problem of collective development from a productionoriented viewpoint. While this view allows a certain amount of anticipatory reflection, e.g., 
the ability for subjects to predict important aspects of development prior to initiation and to assess results after completion (Floyd,1992), it blinds software engineers to the communicative aspects of a collaborative software development activity; (Barthelmess \& Anderson, n.d).

Activity Theory (AT) is a philosophical and cross-disciplinary framework for studying different forms of human practices as development processes, with both individual and social levels interlinked at the same time, (Nardi, 1996).

AT is a development theory that seeks and explains qualitative changes in human practices over time (Uden, n.d). KMS also involves in qualitative changes in human practices, therefore AT is required in the development of KMS. AT helps to maintain adequately the relationship between the individual and social levels (Uden, n.d). KM Systems also incorporates relationship between the managerial personnel and its co-worker to the benefit of the organization (Nardi, 1996). This further emphasizes how AT can play an important role in the development of KM Systems.

AT helps to maintain adequately the relationship between the individual and social levels (Uden, n.d). KM Systems also incorporates relation between the managerial personnel and its co-worker to the benefit of the organization (Stahl, 2006), this emphasizes AT definitely plays an important role in the development of KM Systems.

\subsection{The connection between knowledge management system and software engineering}

KMS is developed in order to convince the need for improving productivity and the potential of employees and the company as a whole. The existing knowledge infrastructure is evaluated so that it can convey the idea that the present ways of doing things are not just sidelined in preference for a new system. KMS is a commercial system, generally IT based, mainly for managing knowledge and experience in organizations supporting creation, capture, storage and dissemination of information (Benbye, 2008).

In essence, $\mathrm{KM}$ is a method that simplifies the process of sharing, distributing, creating, capturing and understanding of a company's knowledge (Davenport \& Prusak, 1998). KM starts with a problem and ends with a solution.

Software Engineering (SE) concerns methods and techniques to develop large software systems. The engineering metaphor is used to emphasize a systematic approach to develop systems that satisfy organizational requirements and constraints. Since SE is a typical knowledge-intensive discipline that evolves very fast and involves a large number of people, different phases and different activities (Rus \& Lindvall, 2002), it is one of the disciplines that can benefits most from KM (Edwards, 2003). Does this mean that KMS follows SE principles?

It can be derived that both practices (KM \& SE) start with a problem. For an example, one of the challenges in KM is to change organizational culture, whereby it involves changing the people attitudes and behaviours to ensure that they contribute to the sharing of knowledge to the organization. However to use SE to develop a large software system in the industry, the processes also follow a number of defined steps which are accepted as best practice by the software engineers. The early phase for SE starts with information or requirements gathering while KMS requires knowledge capture in order to kick-start the development phases. Both the software engineers and the knowledge developer need to specify the suitable tools for designing their intended systems. From this discussion, both practices 
have the same concept, which is problem solving but the approach and the domain may possibly be different. KM involves organizational, human and technical issues, with the advice that the technical should be treated as least important of the three and the most important part of the KMS is to capture experience which is the knowledge itself (Davenport \& Prusak, 1998).

We should see KMS and SE principles differently even though both these practices have the same goal which is to develop a system that help solve problems but the end product of both will not be the same. We use SE principles to develop a conventional system to solve day to day problems such as library system, content management system, point of sales software and other software that are related to our daily use.

The real purpose of the software that has been developed is achieved by looking inside the software as to what does the software or the system contain? Based on simple logic, we can say that the library system contains information about books, names, authors, locations and the ISBN codes and this is what distinguishes a library system from other systems. What then makes a system that is based on SE principles different from KMS?

SE principles may not be sufficient for KMS because to some extent KMS is completely different from a SE system. Both are software, but what goes inside the software might be different as stated earlier. What goes inside the SE system consists of information such as account numbers, stock quantities and others, which are explicit as opposed to KMS, which consist of tacit knowledge which is completely different from information.

Knowledge is distinguished from information by the addition of 'truths, beliefs, perspectives and concepts, judgments and expectations, methodologies and know-how'. Based on opinions, the way we capture information is different than knowledge because knowledge is tacit and from tacit, one can capture experience.

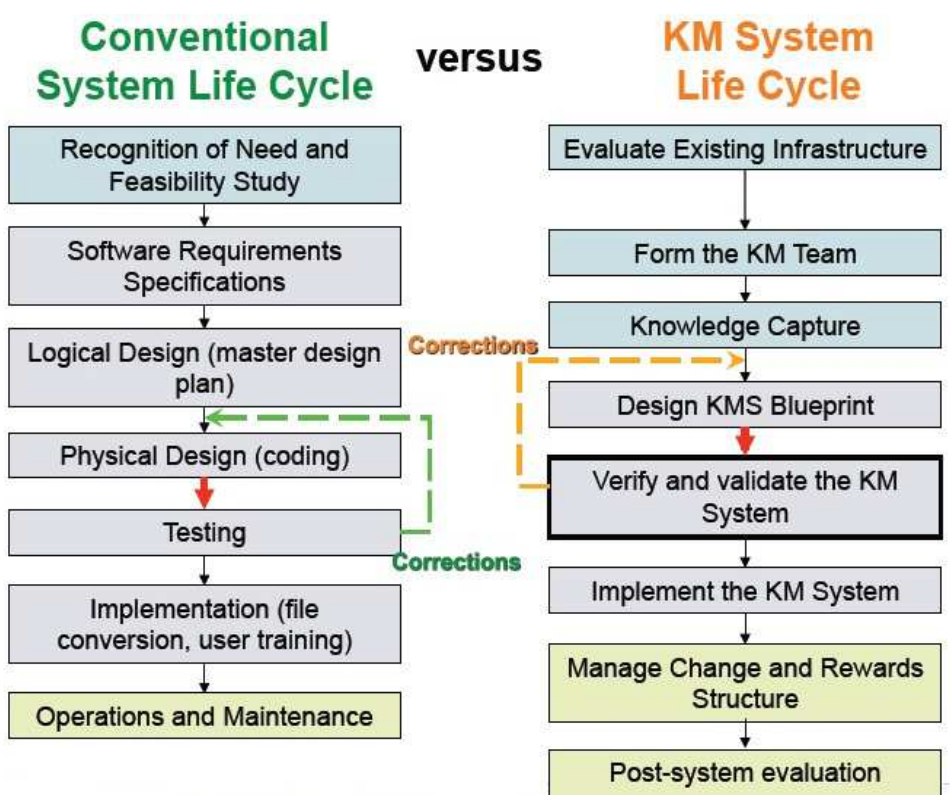

Fig. 1. Adapted from Sornlertlamvanich, V. (Sornlertlamvanich, n.d) 
From the table below, we can see how KM systems differ from conventional systems to warrant a different approach:

\begin{tabular}{|c|c|}
\hline KM System & Conventional System \\
\hline $\begin{array}{c}\text { Knowledge developer gathers knowledge } \\
\text { from people with the given knowledge and } \\
\text { the developer depends on them for the } \\
\text { solution }\end{array}$ & $\begin{array}{c}\text { Systems analyst gathers data and } \\
\text { information from the users and } \\
\text { the users depend on analysts for the } \\
\text { solution }\end{array}$ \\
\hline $\begin{array}{c}\text { Knowledge developer deals with the } \\
\text { domain expert }\end{array}$ & $\begin{array}{c}\text { Conventional system developer deals with } \\
\text { the user }\end{array}$ \\
\hline Experts know the solution and problem & Users know the problem, not solution \\
\hline Result oriented, incremental, interactive & Process driven, sequential \\
\hline Testing phase at the beginning & Testing phase at the end \\
\hline
\end{tabular}

Fig. 2. Adapted from Sornlertlamvanich, V. (Sornlertlamvanich, n.d)

KM team must capture the knowledge requirements for the system to be built so that the end product will be meaningful to the organization and its users. Conventional systems development is primarily sequential, whereas KMS is incremental and interactive. In the case of a conventional system, testing is usually done towards the end of the cycle (after the system has been built), whereas in KMS, the evolving system is verified and validated from the beginning of the cycle. Systems development and systems management is much more extensive for conventional information systems than it is for KMS. The conventional systems life cycle is usually process-driven and documentation-oriented whereas KMS is resultoriented (Sornlertlamvanich, n.d).

\subsection{The weakness of present KMS in achieving its intended goal}

KMS can be a very complex system which comprises of expensive hardware solutions and complex indexed database. When KMS is completed, the developers of the system need to ensure that KMS is able to store tacit knowledge so that their effort proves to be successful. KMS has a reputation for costing a lot to set up, running into difficulties when trying to get everyone to contribute and ending up with a great deal of information that is inconsistent and irrelevant for most of its users (Straker, 2009).

Organizations tend to focus more on the technology used in the development of KMS rather than the requirements or extraction of tacit knowledge to be stored in the KMS. Organizations think that investment in expensive technology can provide them with a good KMS. KM involves organizational, human and technical issues, with the advice that the technical should be treated as least important of the three (Davenport \& Prusak, 1998). Complex IT infrastructures for KMS prove to be costly because the effort need to maintain, update and develop is huge. Instead of focusing too much on the technical view, the developer should focus more on the human part where the KM team should play their role by dealing with the domain experts, experts that know the solution, problem and also can contribute how to make sure the data inside the KMS is tacit knowledge. Knowledge capturing is crucial in developing KMS because before implementing or designing the system, the knowledge has to properly assign to the intended user for future usage.

Failing to capture tacit knowledge will result in the KMS just being a normal database system containing data that is not meaningful for the organization. Tacit knowledge is the 
result of the human brain processing, analyzing and filtering information to reach conclusions. Information is not knowledge. Yet many organizations fail to understand the difference and are disappointed when a huge investment in technology does not deliver the expected results (Hurley, 2010). Organizations need tacit knowledge because it can drive the organization forward and increase the efficiency on how the organization can operate and be more competitive. In an organization, workers come and go and some of them might have ten to fifteen years of working experience. Their invaluable experience in handling certain practices is important for the organization to expand its potential and knowledge capabilities.

Building a system for $\mathrm{KM}$ is thoroughly different from building a system for conventional purpose. As discussed earlier, abandoning social, cultural and organizational issues may interrupt the development process of a good KMS. The character of learning and sharing needs to be cultivated in the foundation of any given organization. Workforces that are willing to learn and share their ideas and experiences in an organization can enable the KMS to perform better. When a knowledge management initiative is seen as the exclusive mandate of the technological department it can become an exercise in information and document storage and retrieval. Successful knowledge management is about fostering an environment in which knowledge is shared and questions asked and answered across the internal barriers of departments and teams.

Most organizations are still structured along hierarchical lines that are not conducive to interdepartmental collaboration or cooperation and yet this collaboration is essential to knowledge management. Finding and managing the flow of knowledge in an organization requires a very different approach to managing information. Creating an organizational culture where knowledge sharing is the norm is the most important and most difficult part of implementing knowledge management within a business. As with all organizational change, technology can and does play an important and integral part but it cannot alone be the driver (Hurley, 2010).

KMS approach without input from all stakeholders can interrupt KMS intended goal. Current knowledge management technologies cannot yet handle uncertainties with inadequate information. They cannot deliver the right information to the right person at the right time because companies cannot predict what the right information to distribute is and who the right people to distribute it to are (Lang, 2001). Stakeholders need to anticipate the prospect of building the KMS and also to identify the experts needed to collaborate with the developers of KMS. Besides, inadequate knowledge or data in the KMS may perhaps be one of the weaknesses of the current KMS available. The quality of information can also be a turn-off. If the first item the user opens is unexpectedly scrappy, then the user will not look further (Straker, 2009). The user's experience is important when navigating the KMS in order to look for specific knowledge. If the experience is not worth a visit due to the poor quality of data stored, then the user might not consider using the system again.

Other than the quality of the data in KMS, a complex user interface and a complex operation can also be an obstacle for the user to use the KMS. When the users only want a simple system and do not have the time to learn how to use it fully then such systems are liable to fall into disuse (Straker, 2009).

Another strong reason why KMS fails in achieving its intended goal is because people are unwilling to cooperate during requirement elicitation for KMS. Apart from that, when the KMS is completed, there is no valuable knowledge input to the KMS itself. People are reluctant to share their invaluable experience and knowledge that they have acquired over 
the years to their organization. They may feel they are 'giving away the shop', deskilling their job and reducing their employability or potential to earn higher wages. Experts, in particular, live and die on what they know. Where knowledge is power, to give freely what you know to others can seem like professional suicide. It may also be work that is rewarded relatively poorly and can lead to people following up and asking you for further information (Straker, 2009).

\subsection{Research gaps}

KM approaches should include methods to overcome impediments to knowledge transfer. Implementing effective methods to counteract impediments in this way may not always be possible (Szulanski, 1996). For example, it may be too much to expect that contributors describe a knowledge artifact, including the factors that associate the strategy with the original context, and how the strategy should change when applied to different contexts.

KM approaches may fail when they attempt to create a monolithic organization memory. Organizations that have tried to develop a massive organization memory as a whole have failed (Ackerman, M. S. and Halverson, C. A. (2000)). Among other reasons, such organizations are distributed and may have conflicting goals.

Such organizations may fail when they do not incorporate with humans, processes, and technology (Ackerman \& Halverson, 2000). This is justified by the limitations and importance of each of these components. Humans alone are slow and have limited capacities. Processes are the main component in delivering organizational goals. Thus, any approach that is not associated with processes will tend to fail or be perceived as failures.

\section{Research method}

Most KMS are so diverse, spanning across industries. In order to obtain necessary data to answer the research question, it is imperative that the research method employed is flexible enough to cater the needs of a variety of situations and to a wide range of complex problems, for which there is often no other suitable means of analysis. The Delphi technique is a systematic method of collecting opinions from a group of experts through a series of questionnaires. The iterative approach espoused in Delphi allows KM experts to reconsider their judgements in the light of feedback from peers. The anonymity of the approach enables experts to express their opinions freely, without institutional loyalties or peer group pressures getting in the way.

The aforementioned iterative approach also gives KM experts more time to think through their ideas before committing themselves to them, leading to a better quality of response. Since AT emphasises on human activity as the basic unit of analysis, the research is able to uses human activities (supervision of projects) to analyse responses garnered from Delphi.

\section{Scenario}

The Five-Year Strategic Plan of University $X$ has made teaching and learning a major priority. Under the said target plan, technology is identified as one of the drivers that will shape the future direction of the University's learning experience. Hence, the gradual adoption of e-Learning tools and strategies is currently being investigated. On that note, the critical success factor and challenges for the implementation of e-Learning at the University is the willingness and ability of existing faculty members to adopt technology into their 
teaching and learning activities. The key thrust is to ensure that lecturers, as front liners, are the ones driving and advocating technology.

Ensuring sustainable buy-in from the academic staff can only be possible when strong structural and technical support is in place. In doing so, tacit knowledge from experts is to be obtained to better comprehend the intended requirements of the tools that would be acquired. To guarantee success in implementation, there must be significant reliance on sound planning and on the provision of appropriate developmental interventions. This would include obtaining tacit knowledge as an input capture into the KMS system from selected experts of best practices, opinions and solutions via the Delphi method.

The process of Delphi mindfully uses the Activity Theory notion in obtaining human related information allowing social-organization processes to be accounted. With this in mind the KMS system would visibly include all needs and demands of the experts including cultural and organizational norm in living up to its expectations and survive the test of time hence productively achieving and solving the software engineering practices of today.

The initial groundwork of the Delphi process taken on for this research study obtained relevant requirements from knowledge experts based on their respective specialization areas. Only five identifiable experts in the cluster of major specialization areas of Information Technology were consulted into providing these relevant feedbacks. These five areas broadly covers the relevant domains in Computing. The major specialization areas include algorithms and network security, multimedia, object oriented programming and game development, databases and software development concepts and strategies.

Upon identifying the experts, the first round of the Delphi process addressed relevant questions directed to the selected experts taking into consideration both the human expert opinions in the subject domain areas together with the social and organizational requirements of the system. They were directed in such a way that the opinions incorporated issue that affected the outside boundary of the organization - the external environment. By driving these questions, the research pilot study enabled the experts to provide opinions that not only drives or shares their current best practices utilized but envisages the possible needs of what makes an outstanding learning Institution.

Further to this, the second round of the Delphi process initiated a set of questions via a questionnaire to the same selected sets of experts. The objective of this stage of the Delphi process is to obtain a consolidated wholesome viewpoint of all experts concerned regarding feasible requirements of the blended learning tools and strategies. A system perspective view on their feedback, opinions and knowledge better enables the complete requirements acquisition process. By doing so, the team is able to obtain a collective consensus in achieving the purpose of the intended change.

\section{The findings}

\subsection{Delphi process step 1 - Outcome}

1. Requirements and effective elicitation results on the blended learning tools.

2. Identifiable assistance that would be required in adopting blended learning.

Expert 1-Algorithms and Computer Security

1. The learning tools should be able to simulate animations via multimedia or games. It should have the features or options to simulate or modelling via a request from expert (lecturer). Choice of simulation should be based on the topic at hand and the guidance 
of the lecturer. The simulation options should have common characters available, hence having the option of choosing the match of which characters are to be adopted or used for the topic or scenario at hand. Simulation via game infuses students to think logically and analytically hence drawing the results via a step-by- step momentum governing a build-up of interest on the topic at hand. A video capture of the lecture with the ability of conversion to audio form provides a thinking flow in the students mind hence mind provoking to empower and challenge the questioning technique. These applications can be converted to any choice of language hence making it multilingual. It supports and garners a cultural openness in assisting students from all walks of life. In addition to this there is a feature of an SMS option linking the lecturer to the student enabling the sharing of instant up-to date information.

2. The need for relevant training from prospective vendors enabling the maximization of the learning tool at hand is required. A testing period performed on a trial basis on the relevant tools should be incorporated to better understand, enable gradual adoption and gauge the capabilities of the software. In the case of minimal support, the success of the software could be jeopardized. It is only feasible through teamwork for a better understanding of the stakeholders of the software.

Expert 2- Multimedia

1. Taking into consideration that this specialization area covers more of the tool manipulation in multiple media format, the provision for recording of visual screen movement enabling the capture of the instructors input entry would be substantial in the display of the software's capabilities. Alongside this would be the creation of a central repository for all visual capture in support of the teaching materials. Again, the provision to support these visual content via narration to a multi-lingual audio content well establishes an open community support culture for international students. The strength of a two-way communication through a discussion forum would enable students to highlight concerns or ideas in the area of module coverage - hence exploring many horizons of skills, capabilities and doubts. Possible inclusion of various on-line games highlighting usage of various media format could also be added as a repository in understanding the "real-life" production conceptualization in a practical way.

2. To consider various vendors who could propose some viable tools that could be adopted into teaching Multimedia using blended strategies in particular the best practices that have been successful in leading institutions that have incorporated blended strategies in their teaching and learning. For that, essential training is required for the adoption of any of the tools that are viable. A considerable time for planning would be required in the rollout of the tools to best enable positive adoption amongst all stakeholders. Facilitators should be equipped with financial support in acquiring some state-of-the art technological tool to better support the upgrade of the technological adoption in the institution.

Expert 3 - object oriented programming and game development

1. One blended tool that could be incorporated should enable programming to be visually guided (screen view) as video captured files which could be made 
available publicly via YouTube. This would be possible with screen capture software highlighting the visual movement of the input device hence enabling the students to revisit lectures. The capture of the lecture (visual input screen movement) could be utilized as a revision through examples given or perhaps be used as an alternative mode of teaching. Simulation depicting object oriented programming principles would also be viable to better understand the topic at hand. We could also use gaming software's which would strengthen the knowledge of students via a self -guided realization. Another feature that could be added in the imperative technology of making communication effective both ways is to enable the blended learning tool available via mobile applications. The objective here is mobility of being able to gain access to the expert (lecturer) anytime and anywhere. Students and lecturers at the University are respectively logged on the system. This could be featured as a Universities "Facebook" application. Another interesting requirement is acquiring tools that enable group collaboration that logs problems and shares solutions with individuals and or groups thus enabling feedback from lecturers to be appropriately provided. The additional feature of the blended tool to enable on-line video conferencing with students and potential speakers and clients could also carve a niche in overcoming the communication and requirement gap.

2. There is a need for vendors support on the software's adopted. Training is essential on the best technical video capture (awareness of all possible tools and methods of video or audio capture) in adopting the right blended learning strategy. Quality of tool is essential. It is equally important to ensure that the content of the video is appropriately suitable for the nature of the study or practice in accordance with the current Gen Y. It also provides support to experts looking for the best simulators available in teaching object oriented programming at a higher level other than ALICE. There is a visible need for up to date technological gadgets and tools with the objective of keeping up with the current trends hence making it appealing to try out new techniques and styles of blended learning. Recognition and reward should be given to individual experts in taking the initiative to appropriately apply blended learning into their classroom. This serves as an internal and external motivational factor to many.

Expert 4 - Databases

1. There should visibly be simulation of the programming of SQL through a recorded session thus enabling students to conceptualize the practical sessions through a simulation learning system, serving as a recap or perhaps a revision of the topic at hand. The need to establish an online forum to ensure that the students are able to interact with lecturers who serve as a support mechanism to students and lecturers enabling better understanding of topics and ideas discussed. Example: freeforum.org. There should also be access to video files depicting real life industry case studies to be viewed by the students for practical experience and analysis. Students need to be provided with real-time data which could be used to train the students on database development for high end industries.

2. There is visible need for trainings from the suppliers or vendors on the usage of the specific product. Lecturers should be able to gain access to up-to-date resource 
materials through the University's library. Financial support into the investment on technological gadgets such as Ipad could develop a new culture towards the ecosystem of the University.

Expert 5 - Software development concepts and strategies.

1. The tools should enable facilitated collaboration with industry stakeholders thus providing an avenue for direct interaction of problem and solutions. How can this be done? Via a forum that could automatically send out "invites" to industry experts. This would be a specialized tool that caters to the requirements of round trip engineering (software). The tools should contain a database, self- available samples of past models or solutions with feedback and comments attached to each, hence becoming a repository of knowledge base. Inside the repository would be viable and non-viable solutions.

2. The need for collaboration with vendors is crucial in building the intended applications best suiting the user at hand. Clientele assistance is needed in identifying committed clients who are willing (via a Memorandum of Understanding) to be pro-actively involved in injecting real-life industry inputs. There is a need for assistance in identifying real-life projects to be used for assignments. The repository has to be current and updated with solutions for future reference and commercialization purposes (if required).

\section{The interview process}

The goal of this questionnaire process at the end is to reach a consensus of opinion among the group of experts. The uniqueness of Delphi lies in its reliability, given the variableness of human opinion, and in its ability to be administered remotely without direct participant interaction. Hence, this process gathers a reconfirmation of the experts' knowledge and opinions on forming the basis of a collective organizational goal that is to be achieved. The individual experts will be given a brief explanation of the selection options, criteria settings which will then guide them to provide their opinions.

Questions:

1. What would you want in a tool to assist you in incorporating Blended Learning in or outside classrooms?

2. The effectiveness of the tools based on subject domain?

3. What assistance would you as an expert require in adopting Blended learning?

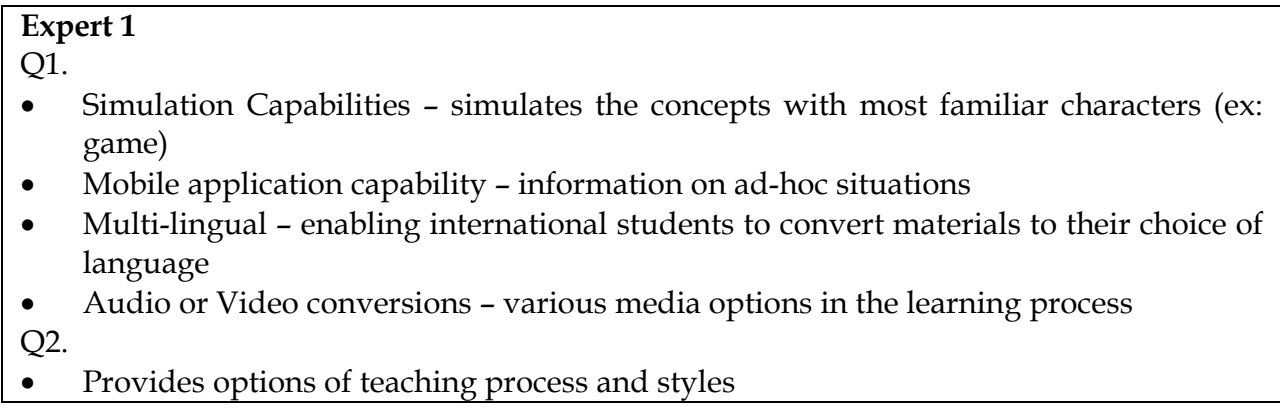




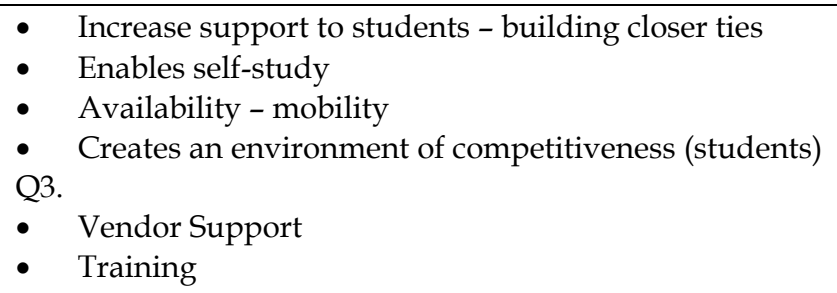

\section{Expert 2}

Q1.

- Simulation capabilities - make it interesting

- Mobile application capability - fast accessibility to lecturers

- Multi-lingual - to assist international students as a supporting application

- Visual screen capture - to be stored in repository for reference

- Real-time data capture

- User friendly

Q2

- Enables self-study

- Available - mobility

- Improves logical thinking process - via games or simulations

- $\quad$ Builds interest - current technology trends being practiced - keeping up with IT savvy skills of students

Q3

- Management support

- Training

- Technology devices - current trends - Ipad

\section{Expert 3}

Q1

- Simulation capabilities - able to help simulate programming topics

- Mobile application capability - making communication effective

- Visual screen capture - to capture screen of programming process

- Forum - all stakeholders - industry based online forum where we assist industry personnel's in given advice and solutions

- Games simulation capabilities - enabling better understanding of programming

Q2 principles

- $\quad$ Provides options of teaching process - different from traditional method of teaching using technology as an aided tool

- Increases student support - building closer ties - anywhere, anytime concept

- Enables self-study - encourages independent study of student

- Available - mobility - accessing the materials becomes easily accessible

- Engaging in industrial relation or ties - real experience gained on real work scenarios

- Improves logical thinking process via games or simulations - logic approaches simplified. 


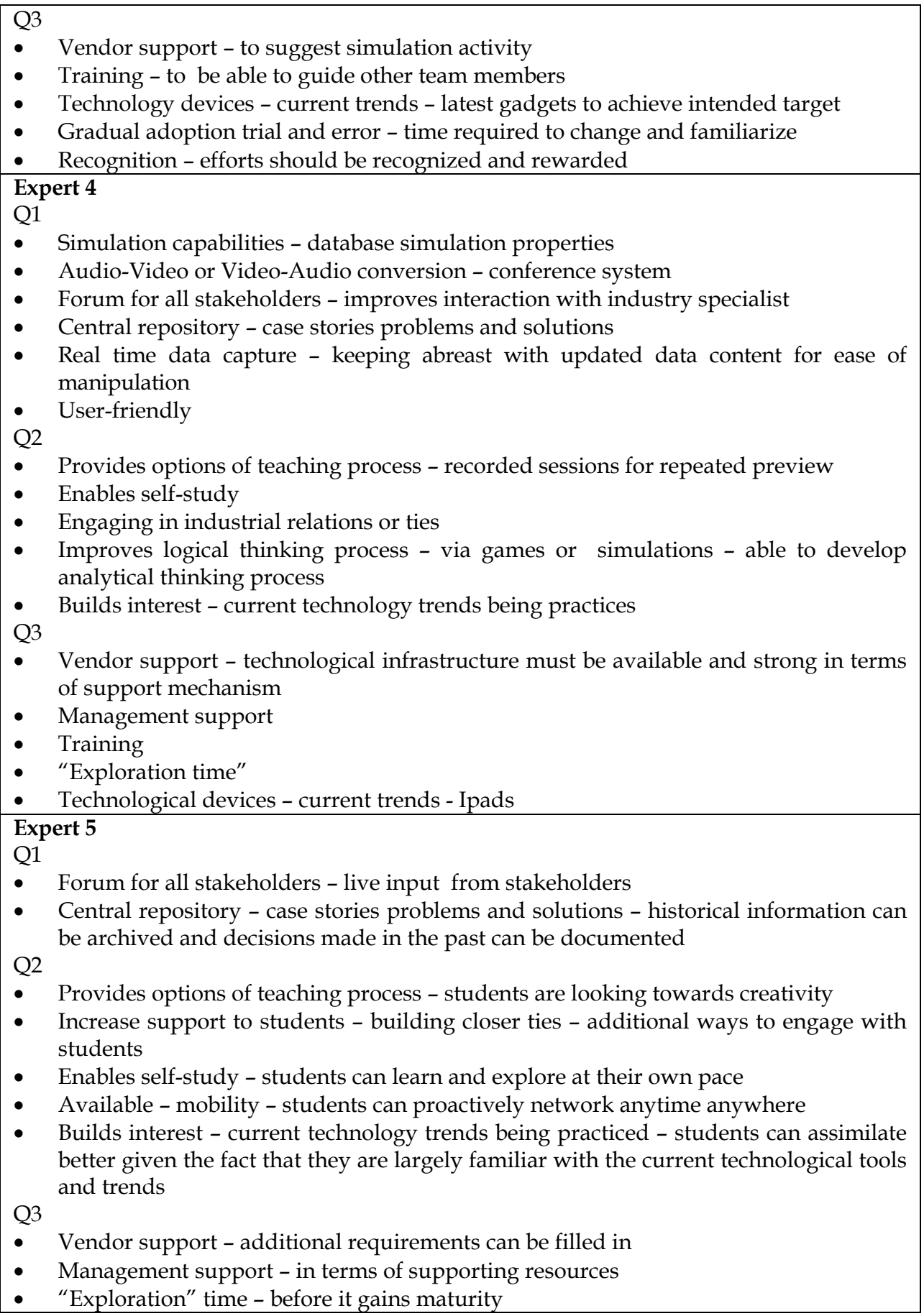




\section{Analysis of findings and conclusion}

The success of any KMS lies in the effective input of knowledge from all stakeholders concerned. Knowledge, which transforms itself as requirements into the KMS system, has to evidently involve proactive input from experts. These experts are those who understand the organization's processes and culture better and thus able to provide valuable and imperative input. Hence, one way of analysing this human activity occurrence serving as tacit knowledge into the KMS system is by using the Activity Theory. Since AT focuses on the interaction of human activity and consciousness within its relevant environmental context, the eventual KMS developed around these fundamentals would have an achievable and sustainable success rate of enabling employees to seamlessly access the organization's documented facts, best practices and solutions.

The Delphi technique utilized in this research study involved firstly gathering opinions and knowledge from selected experts concerned. The process went through a second round that led to a re-confirmation of opinions and knowledge serving as a building block for the solutions at hand. The research concluded from the Delphi process documented above evidently shows common opinions and knowledge that were gathered from the experts. It is apparent that all experts are in agreement for a blended learning initiative with the support and involvement from all stakeholders concerned.

By using both activity theory and Delphi technique, deep analysis can be performed within the activities of the experts and from these activities, tacit and explicit knowledge can be observed and analyzed. During the interview process, especially during the second round of interviews, when the questions were asked to the panel of experts, some of the experts realized that there existed divergent approaches exist in supervision style. Not only was the knowledge engineer able to garner further insights, the exercise also proved by an eyeopener for experts themselves which would have inadvertently increased their body of knowledge.

As espoused in this research, software engineering practice generally does not amalgamate people, cultural and organizations factors when gathering requirements. Since it is imperative that KM Systems consider the aforementioned factors, AT coupled with Delphi technique was applied in this research. This approach is not only holistic but also more dynamic with thorough investigations built in to the roadmap involving people, cultural, and organization dimension. The investigations details how using AT, a knowledge engineer is able to harvest tacit knowledge allowing every situation and scenes to evolve gives them different meaning and context. In conclusion, this research espouses a combination of KM practices, AT and Delphi technique in an integrated roadmap to provide a solid foundation for requirements elicitation in developing KM system.

\section{Research outcome and limitations}

The proposed research will shed new insights on the roadmap required to implement an effective KMS that will survive the test of time. The roadmap will ensure that KMS maps the interest of its stakeholders alike since it encapsulates their interest from the onset. The roadmap embodies an interesting fusion of people, cultural and organizational aspects required of a successful KMS.

However, it must be noted that even when the aforementioned system is in place, it still does not eliminate the perennial challenge of most knowledge management systems in place 
today which is the aspect of contribution. If contributions are not forthcoming, then even the most illustrious KMS will not serve its intended purpose. The proposed KMS roadmap does not illustrate a detailed outline of the development processes required to implement a KMS but rather elicitation of requirements required as building blocks to build a KMS.

\section{References}

Abecker, A., Decker, S. and Maurer, F. (2000) 'Organizational Memory and Knowledge Management', Guest editorial, Information Systems Frontiers, vol. 2, no. 3-4, pp. 251-252.

Ackerman, M. S. and Halverson, C. A. (2000) 'Reexamining Organizational Memory', Communications of the ACM, vol. 43, no. 1, pp. 59-64.

Benbye, H (2008). Knowledge Management Systems Implementation: Lessons from the Valley. Oxford, Chandos Publishing.

Davenport, T.H. and Prusak, L. (1998), Working Knowledge: How Organizations Manage what they Know, Harvard Business School Press, Boston, MA.

Floyd, C.: 1992, 'Software Development as Reality Construction'. In: C. Floyd, H. Zullighoven, R. Budde, and R. Keil-Slawik (eds.): Software Development and Reality Construction. Springer-Verlag, Chapt. 3.2.

Hurley.L (2010) Reasons knowledge management information systems fail (n.d.).

I. Rus, and M. Lindvall, "Knowledge management in software engineering," IEEE Software, vol. 19(3), 2002, pp. 26-38.

J. S. Edwards, "Managing software engineers and their knowledge," in Managing Software Engineering Knowledge, A. Aurum, R. Jeffery, C. Wohlin, and M. Handzic, Eds., Berlin: Springer-Verlag, 2003, pp. 5-27.

Lang, J. C. (2001). Managerial concerns in knowledge management. Journal of Knowledge Management, 5(1), 43-59. doi:10.1108 or 13673270110384392

Langton, N \& Robbins, S. (2006). Organizational Behaviour (Fourth Canadian Edition). Toronto, Ontario: Pearson Prentice Hall.

Lee, H. and Choi, B., Knowledge Management Enablers, Processes, and Organizational Performance: An Integrative View and Empirical Examination, Journal of Management Information Systems, Volume 20, Number 1, 2003, pp. 179 - 228.

Nardi, B., Ed. Context and Consciousness: Activity Theory and Human-Computer Interaction.Cambridge, MA, MIT Press, 1996.

P. Barthelmess and K.M. Anderson University of Colorado at Boulder A View of Software Development Environments Based on Activity Theory

Sornlertlamvanich, V. (n.d.). 2: Knowledge Management Systems Life Cycle. Knowledge Management.

Stahl, G. (2006), Group cognition: Computer Support for Building Collaborative Knowledge, MIT Press, Cambridge, MA.

Straker, D. (2009). How Knowledge Management Systems Fail and Succeed. syque.com. Syque.

Szulanski, G. (1996) 'Exploring Internal Stickiness: Impediments to the Transfer of Best Practice within Firms', Strategic Management Journal, vol. 17, Special Issue Winter, pp. 27-44.

Terra, J.C. \& Gordon, C. (2002) Realizing the promise of corporate portals: Leveraging knowledge for business success, Boston: Butterworth Heinemann 
Uden, Lorna Department of Computing, Engineering and Technology, Staffordshire University, Beaconside, Stafford, ST18 0AD, UK E-mail: 1.uden@staffs.ac.uk Activity theory for designing mobile learning 


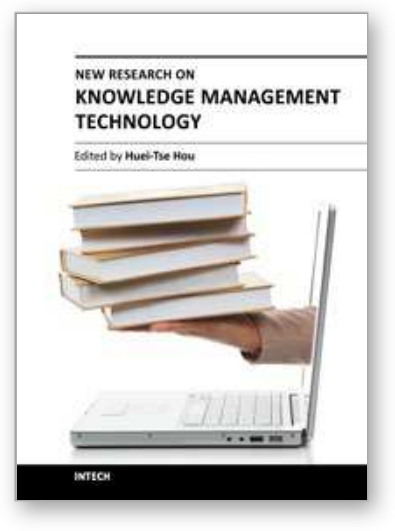

\author{
New Research on Knowledge Management Technology \\ Edited by Dr. Huei Tse Hou
}

ISBN 978-953-51-0074-4

Hard cover, 228 pages

Publisher InTech

Published online 24, February, 2012

Published in print edition February, 2012

Due to the development of mobile and Web 2.0 technology, knowledge transfer, storage and retrieval have become much more rapid. In recent years, there have been more and more new and interesting findings in the research field of knowledge management. This book aims to introduce readers to the recent research topics, it is titled "New Research on Knowledge Management Technology" and includes 13 chapters. In this book, new $\mathrm{KM}$ technologies and systems are proposed, the applications and potential of all KM technologies are explored and discussed. It is expected that this book provides relevant information about new research trends in comprehensive and novel knowledge management studies, and that it serves as an important resource for researchers, teachers and students, and for the development of practices in the knowledge management field.

\title{
How to reference
}

In order to correctly reference this scholarly work, feel free to copy and paste the following:

Sanath Sukumaran, Hemalatha Ramalingam, Lorna Uden, Akmal Rahim and Kanchana Chandran (2012). A Roadmap for Requirements Elicitation of Knowledge Management Systems: A Delphi Study, New Research on Knowledge Management Technology, Dr. Huei Tse Hou (Ed.), ISBN: 978-953-51-0074-4, InTech, Available from: http://www.intechopen.com/books/new-research-on-knowledge-management-technology/a-roadmap-forrequirements-elicitation-of-knowledge-management-systems-a-delphi-study

\section{INTECH}

open science | open minds

\author{
InTech Europe \\ University Campus STeP Ri \\ Slavka Krautzeka 83/A \\ 51000 Rijeka, Croatia \\ Phone: +385 (51) 770447 \\ Fax: +385 (51) 686166 \\ www.intechopen.com
}

\author{
InTech China \\ Unit 405, Office Block, Hotel Equatorial Shanghai \\ No.65, Yan An Road (West), Shanghai, 200040, China \\ 中国上海市延安西路65号上海国际贵都大饭店办公楼405单元 \\ Phone: +86-21-62489820 \\ Fax: $+86-21-62489821$
}


(C) 2012 The Author(s). Licensee IntechOpen. This is an open access article distributed under the terms of the Creative Commons Attribution 3.0 License, which permits unrestricted use, distribution, and reproduction in any medium, provided the original work is properly cited. 